\title{
Intravenous magnesium sulfate for acute bronchiolitis: evaluation of the effect on clinical course and outcomes
}

\author{
Nihan Şık ${ }^{1}$, Hale Çitlenbik ${ }^{2}$, Ali Öztürk ${ }^{1}$, Durgül Yılmaz ${ }^{2}$, and Murat Duman ${ }^{1}$ \\ ${ }^{1}$ Dokuz Eylul University Faculty of Medicine \\ ${ }^{2}$ Dokuz Eylul Universitesi Hastanesi
}

February 22, 2021

\begin{abstract}
Background: The aim of this study was to assess the efficacy of intravenous (IV) magnesium sulfate (MgSO4) on clinical severity scores, need for respiratory support and outcomes for previously healthy children with bronchiolitis. Methods: We retrospectively assessed children with moderate-severe bronchiolitis. Patients who received $40 \mathrm{mg} / \mathrm{kg} / \mathrm{dose}$ of IV MgSO4 (group 1) or not (group 2) were compared for vital signs, clinical findings and outcomes. Results: There were 74 patients in group 1 and 33 in group 2. The median age, the mean respiratory rates, oxygen saturation/fraction of inspired oxygen (S/F) ratios and, The Modified Respiratory Distress Assessment Instrument (mRDAI) scores at the time of starting MgSO4 treatment were similar for two groups. Respiratory rate and mRDAI score significantly decreased at the 2th hour of MgSO4 treatment and the decrease was observed for 4 th, 8 th and 12 th hours, compared with group 2. Patients in group 1 had a higher S/F ratio at 4th hour compared with group 2 and in group 1, the elevation was observed at the 4 th hour. Patients in group 2 had a higher rate of requirement and an earlier start high flow nasal cannula oxygen therapy but the total duration time was similar for two groups. Patients in group 2 had a longer hospital stay than group 1. Conclusion: Intravenous MgSO4 provided significant improvement on clinical severity, early phase of oxygenation, need for respiratory support, length of stay in the hospital and outcomes. It seems to be an effective treatment option for management of bronchiolitis.
\end{abstract}

Intravenous magnesium sulfate for acute bronchiolitis: evaluation of the effect on clinical course and outcomes

Nihan Şık, MD ${ }^{1}$, Hale Çitlenbik, MD ${ }^{1}$, Ali Öztürk, MD ${ }^{1}$, Durgül Yılmaz, Prof. ${ }^{1}$, Murat Duman, Prof. ${ }^{1}$

${ }^{1}$ Division of Pediatric Emergency Care, Department of Pediatrics, Dokuz Eylul University, Faculty of Medicine, Izmir, Turkey.

Corresponding author: Prof. Dr. Murat Duman, Division of Pediatric Emergency Care, Department of Pediatrics, Dokuz Eylul University, Faculty of Medicine, Izmir, Turkey. e-mail:mduman@deu.edu.tr, Tel: +902324126015

Keywords: Acute bronchiolitis, children, magnesium sulfate

\section{Funding}

This research did not receive any specific grant from funding agencies in the public, commercial, or not-forprofit sectors.

\section{Declaration of Conflicting of Interests}

The authors declare no conflict of interest. 
This study was presented as an oral presentation in XVI. Pediatric Emergency Medicine and Intensive Care Congress.

Running head: Intravenous magnesium sulfate for acute bronchiolitis

\section{INTRODUCTION}

Acute bronchiolitis $(\mathrm{AB})$ is an infection of the lower respiratory tract that caused by viral agents, especially respiratory syncytial virus, most prevalent in children aged less than 24 months. It is the most common reason for hospital admissions in the first year of life, representing a significant health burden worldwide. Bronchiolitis usually demonstrates a benign course, most patients are treated as outpatients but progression to severe illness may occur rapidly and respiratory support and admission to intensive care unit may be required promptly in some cases ${ }^{1}$. It is characterized by damage of epithelial cells leading ciliary destruction, airway inflammation, edema and increased mucus production. Mucus plugs and cellular debris cause obstruction of bronchiolar lumens and result in various degrees of respiratory distress ${ }^{2}$.

Current recommendations for treatment of $\mathrm{AB}$ focus on supportive care, including respiratory support, oxygen supplementation if needed and adequate hydration. Other treatment agents such as bronchodilators, hypertonic saline, corticosteroids, antiviral/antibacterial agents showed no clearly defined benefit. Only high flow nasal cannula (HFNC) oxygen therapy has been elucidated as a new and promising tool for treatment ${ }^{\mathbf{3}_{-}}$ 10 .

Magnesium is an essential cofactor of many enzymatic reactions. It acts as a regulator of calcium translocation across cell membranes, blocking N-methyl-D-aspartate receptor-gated calcium channels; blocks calcium influx into the smooth muscle cells. As a result, magnesium inhibits the contraction of smooth muscle and has a bronchodilator effect on respiratory system. It inhibits the acetylcholine and histamine release from cholinergic nerve terminals and mast cells and decreases glandular mucus production. Magnesium also attenuates neutrophil respiratory burst, as having a role in regulation of anti-inflammatory processes ${ }^{11}{ }^{13}$.

Intravenous (IV) and nebulized magnesium sulfate (MgSO4) treatment has been used for management of adult and pediatric bronchial asthma. In pediatric population, IV MgSO4 was found to safely improve bronchoconstriction, pulmonary function and reduced hospital admission. Nebulized MgSO4 was concluded to result in modest additional benefits when used as an additional treatment to inhaled $\beta 2$-agonists and ipratropium bromide although evidence was low and there remained substantial uncertainty ${ }^{1} 4^{-1}{ }^{1}$.

Magnesium sulfate was also investigated as a treatment option for bronchiolitis in very few studies. To our knowledge, there were 2 studies evaluating nebulized and one study IV high dose of MgSO4 use for bronchiolitis patients ${ }^{1} 8_{-}^{20}$. The aim of this study was to assess the efficacy of IV MgSO4 treatment on clinical severity scores, oxygenation, need for respiratory support and outcomes for previously healthy moderatesevere bronchiolitis patients.

\section{MATERIALS AND METHODS}

\section{Study design}

This was a retrospective cohort study of infants with bronchiolitis that performed at the Dokuz Eylul University Pediatric Emergency Department, having approximately 100.000 admissions annually, between December 2018-May 2019. Before starting the study, the ethical approval was obtained from the local ethics commitee (approval number: 2020/01-01). According to the American Academy of Pediatrics guidelines, previously healthy children diagnosed with acute bronchiolitis aged between 1-24 months were eligible for the study4. We searched patients from computer database and obtained information from electronic medical records and nursing records. Children with moderate and severe bronchiolitis according to The Modified Respiratory Distress Assessment Instrument (mRDAI) score (5-12) were included ${ }^{21}$. This scoring system is a scale that consists of respiratory rate, use of accessory muscles, auscultation findings and color; scoring each parameter 0 to 3 and categorizing the total score as follows: 0-4: Mild, 5-8: Moderate and: 9-12: Severe. Patients with life-threatening disesase requiring immediate intervention, having chronic respiratory, cardiac, 
neuromuscular diseases, genetic disorders, immun deficiencies, a history of prematurity $(<34$ gestational week), recurrent wheezing (more than 3 attacks) and a history of a bronchodilator or corticosteroid use within 48 hours of admission and incomplete data were excluded. We also excluded patients who met one major or two minor criterias according to the Modified Asthma Predictive Index in order to minimize the number of patients that had a risk of bronchial asthma ${ }^{22}$. According to the treatment protocol applied in our hospital, patients were given 3 doses of salbutamol nebules with 20 minutes intervals, and if there was a response to the treatment, then it was repeated at 2-4 hours intervals, according to clinical findings. If there was still no response, $1 \mathrm{mg} / \mathrm{kg} /$ dose of methylprednisolone was given. Also, a $6 \mathrm{~L} / \mathrm{min}$ oxygen by a simple face mask was administered anytime if oxygen saturation $(\mathrm{SpO} 2)<90 \%$. In the presence of unresponsiveness to all these attempts, administering a $40 \mathrm{mg} / \mathrm{kg} /$ dose of IV MgSO4 was not standardized in our local protocol, the decision was reserved for the judgement of the clinician. So, children who met our local treatment protocol for $\mathrm{AB}$ were divided into two groups: Patients that received $40 \mathrm{mg} / \mathrm{kg} / \mathrm{dose}$ of IV MgSO4 in 50-100 mL of $0.9 \%$ saline (max:2 gr/day) in 30 minutes as group 1 and patients not received MgSO4 but had similar clinical severity scores as group 2. The demographic data, heart rate, respiratory rate (RR), SpO2, blood pressure, body temperature, fraction of inspired oxygen (FiO2), SpO2/ FiO2 (S/F) ratio, mRDAI scores at $0,1,2,4,8,12$ th hours, clinical findings, laboratory data, medications, need for respiratory support, need for intensive care unit admission, total length of stay in the hospital and observed side effects of MgSO4 (hypotension, arrythmia, loss of deep tendon reflexes, nausea, vomiting) were recorded for each patient.

\section{Statistical analysis}

All statistics were performed by using SPSS software version 22.0 (IBM Cop., Armonk, NY, USA). Data were presented as means with standard deviations (SD) or medians with interquartile ranges (IQRs) and 25th75th percentiles. To compare group 1 and 2, the Mann-Whitney $\mathrm{U}$ test was used to analyze non-parametric variables and Student's $t$ test and one-way analysis of variance (ANOVA) test for parametric data. The paired sample $t$-test and the repeated measure ANOVA, followed by post hoc Tukey test were used to evaluate changes of variables in two groups. $P$ values less than 0.05 were considered statistically significant.

\section{RESULTS}

Among 140 children, 107 of them met all criteria to be eligible for the study. Sixty-nine $(62.7 \%)$ of the patients were male. The median age was 9.0 months (IQR: 4.0-13.0). Of the 107 children in the study, 46 $(49.8 \%)$ of them required for respiratory support. All of them were treated with HFNC oxygen therapy; there was no use of continuous positive airway pressure, bilevel airway positive airway pressure treatments or invasive mechanical ventilation. No patient was admitted to the pediatric intensive care unit; children who required for HFNC oxygen therapy were admitted to the ward and if not possible, they were observed in the observation unit of the pediatric emergency department during the study period.

There were $74(69.2 \%)$ patients in group 1 and $33(30.8 \%)$ patients in group 2 . The median age $(p: 0.296)$, gender ratios $(p: 0.531)$, the mean respiratory rates $(p: 0.424), \mathrm{S} / \mathrm{F}$ ratios $(p: 0.234)$, mRDAI scores $(p: 0.260)$ at the time of starting MgSO4 treatment were similar for these two groups (Table 1). For the patients in group 1, RR and mRDAI score significantly decreased at the 2th hour of MgSO4 treatment $(p$ : $0,018, p: 0,002)$ and the decrease was observed for 4th $(p<0,001)$, 8th $(p: 0,039, p<0,001)$ and 12th $(p: 0,02, p$ $: 0,002$ ) hours, compared with group 2, respectively (Figure 1 and 2). In group 1, the significant decrease of respiratory rate and mRDAI score was seen at the 2 th hour of MgSO4 treatment $(p<0,001)$ and also continued until the 12th hour. Patients in group 1 had a higher S/F ratio at 4th hour MgSO4 treatment compared with group $2(p: 0.007)$ and in group 1 , the elevation was also observed at the 4th hour $(p: 0.007)$ (Figure 3).

The rate of requirement for HFNC oxygen therapy was significantly higher in patients in group 2, respectively. (17.5\% and $36.3 \%, p: 0.035)$. Patients in group 2 had an earlier start of HFNC oxygen therapy $(p: 0.004)$ but the total duration time was similar for two groups $(p: 0.582)$ [the median time at starting HFNC oxygen therapy after admission to the pediatric emergency department:17.6 h (3.0-51.0) for group 1, 9.5 h (2.0-49.0) for group 2; the median duration time: $83.0 \mathrm{~h}$ (10.0-120.0) for group 1, $71.5 \mathrm{~h}$ (24.0-120.0) in group 2]. 
Furthermore, the median length of stay in the hospital was longer in patients in group 2 [58.5 h (10.0-201.0) for group 1 and $77.1 \mathrm{~h}$ (15.0-179.0) for group 2, $p: 0.022)$. No side effect was reported during MgSO4 infusion period.

\section{DISCUSSION}

In the current study, we aimed to investigate the efficacy of IV MgSO4 treatment on clinical severity, oxygenation parameters, need for respiratory support and outcomes in bronchiolitis.

Intravenous and nebulized MgSO4 were assessed for the management of the children with bronchiolitis but there are limited data in the literature; we found two studies evaluating nebulized and one study intravenous high dose of $\mathrm{MgSO} 4$ use for bronchiolitis patients ${ }^{1} 8^{2}{ }^{20}$. Both intravenous and nebulized MgSO 4 treatments were found to have no effect on hospital admissions, unfortunately.

Modaresi et al. evaluated 120 moderate-severe bronchiolitis patients and compared nebulized epinephrine and nebulized MgSO4 plus epinefrine ${ }^{1} 8$. They excluded the patients with a history of asthma in the family and received bronchodilator or corticosteroid use before enrollment. Respiratory Distress Assessment İnstrument (RDAI) score was used to quantify the clinical severity. They found no difference on the first day but RDAI scores improved on the second and third day favoring nebulized magnesium plus epinephrine. The requirement for oxygene or respiratory support was similar and there was no difference in the length of hospital stay between two groups. In another study including 56 children with bronchiolitis, patients were divided into three groups and received nebulized salbutamol with normal saline, magnesium sulfate with normal saline or and salbutamol plus magnesium sulfate. No side effects were observed. Clinical severity scores were recorded at 0,1 and 4 th hours and magnesium sulfate plus salbutamol group had lower clinical severity scores at 4 th $\mathrm{h}$ compared with the other two groups. However, the length of stay in the hospital was similar ${ }^{1} 9$. The amount of MgSO4 delivered by nebulization was stated uncertain in previous studies ${ }^{23}$.

There is only one study investigating the efficacy of intravenous $\mathrm{MgSO} 4$ treatment for bronchiolitis. Alansari et al. evaluated 162 moderate-severe bronchiolitis patients and compared a $100 \mathrm{mg} / \mathrm{kg}$ dose of IV MgSO4 and placebo, a higher dose than we used as $40 \mathrm{mg} / \mathrm{kg}$. They used nebulized epinephrine with $5 \%$ hypertonic saline at $4 \mathrm{~h}$ intervals for all patients and oral dexamethasone for the patients that had a history of eczema and/or a family history of asthma ${ }^{20}$. They concluded that Wang bronchiolitis severity scores and time to readiness for discharge were similar for patients that received MgSO4 or not, contrary to our findings. In both groups, half of the patients had a history of eczema and/or a family history of asthma. The ratio of the groups involving children with a risk of asthma was similar but to differentiate how many of these cases might actually be asthma and benefited from $\mathrm{MgSO} 4$ or dexamethasone therapy was difficult for two groups. This discrepancy may be related to the main efficacy of $\mathrm{MgSO} 4$ not being exposed.

In the current study, we excluded patients who met the criterias to have a risk of bronchial asthma in order to minimize confusion. This might help us assess the effect of MgSO4 more objectively for bronchiolitis patients as we found that children who received MgSO4 had lower respiratory rates and mRDAI scores from the 2th hour and elevated S/F ratios at the 4th hour. Magnesium sulfate decreased the requirement for respiratory support and also shortened the length of stay in the hospital. No side effect of MgSO4 was reported in both these studies, respectively. To our knowledge, this is the first study evaluating the IV MgSO4 therapy on previously healthy bronchiolitis patients that have a low risk for bronchial asthma.

Our study has some limitations. First, we performed a retrospective study and it was conducted at a singlecenter pediatric emergency department; there was a limited number of patients to met all criterias to enroll. Second, cost reports of all medications, HFNC oxygen therapies or hospital stays were not calculated, so we could not compare the cost-effectiveness for patients who received MgSO4 or not.

In conclusion, IV MgSO4 provided significant improvement on clinical severity, early phase of oxygenation, need for respiratory support, length of stay in the hospital and outcomes with no side effects. From this point of view, it seems to be an effective treatment option for management of bronchiolitis. Nevertheless, multicenter studies with larger case series are required to reveal the benefits of IV MgSO4 in children with 
moderate-severe bronchiolitis and to take part in the treatment guidelines.

\section{REFERENCES}

1. Ralston S, Comick A, Nichols E, Parker D, Lanter P. Effectiveness of quality improvement in hospitalization for bronchiolitis: a systematic review. Pediatrics. 2014 Sep;134(3):571-81.

2. Zhang L, Gunther CB, Franco OS, Klassen TP. Nebulised hypertonic saline solution for acute bronchiolitis in infants. Cochrane Database Syst Rev. 2017 Dec 21;12:CD006458.

3. Kyler KE, McCulloh RJ. Current Concepts in the Evaluation and Management of Bronchiolitis. Infect Dis Clin North Am. 2018 Mar;32(1):35-45.

4. Ralston SL, Lieberthal AS, Meissner HC, Alverson BK, Baley JE, Gadomski AM, Johnson DW, Light MJ, Maraqa NF, Mendonca EA, et al. Clinical practice guideline: the diagnosis, management, and prevention of bronchiolitis. Pediatrics. 2014 Nov;134(5):e1474-502.

5. Florin TA, Plint AC, Zorc JJ. Viral bronchiolitis. Lancet. 2017 Jan 14;389(10065):211-224.

6. Korppi M. Therapeutic strategies for pediatric bronchiolitis. Expert Rev Respir Med. 2019 Jan;13(1):95-103.

7. Kou M, Hwang V, Ramkellawan N. Bronchiolitis: From Practice Guideline to Clinical Practice. Emerg Med Clin North Am. 2018 May;36(2):275-286.

8. Flores P, Mendes AL, Neto AS. A randomized trial of nebulized $3 \%$ hypertonic saline with salbutamol in the treatment of acute bronchiolitis in hospitalized infants. Pediatr Pulmonol. 2016 Apr;51(4):418-25.

9. Zhang L, Gunther CB, Franco OS, Klassen TP. Impact of hypertonic saline on hospitalization rate in infants with acute bronchiolitis: A meta-analysis. Pediatr Pulmonol. 2018 Aug;53(8):1089-1095.

10. Castro-Rodriguez JA, Rodriguez-Martinez CE, Sossa-Briceño MP. Principal findings of systematic reviews for the management of acute bronchiolitis in children. Paediatr Respir Rev. 2015 Sep;16(4):26775.

11. Cairns CB, Kraft M. Magnesium attenuates the neutrophil respiratory burst in adult asthmatic patients. Acad Emerg Med. 1996 Dec;3(12):1093-7.

12. Gourgoulianis KI, Chatziparasidis G, Chatziefthimiou A, Molyvdas PA. Magnesium as a relaxing factor of airway smooth muscles. J Aerosol Med. 2001 Fall;14(3):301-7.

13. Blitz M, Blitz S, Hughes R, Diner B, Beasley R, Knopp J, Rowe BH. Aerosolized magnesium sulfate for acute asthma: a systematic review. Chest. 2005 Jul;128(1):337-44.

14. Irazuzta JE, Chiriboga N. Magnesium sulfate infusion for acute asthma in the emergency department. J Pediatr (Rio J). 2017 Nov - Dec;93 Suppl 1:19-25.

15. Kew KM, Kirtchuk L, Michell CI. Intravenous magnesium sulfate for treating adults with acute asthma in the emergency department. Cochrane Database Syst Rev. 2014 May 28;(5):CD010909.

16. Knightly R, Milan SJ, Hughes R, Knopp-Sihota JA, Rowe BH, Normansell R, Powell C. Inhaled magnesium sulfate in the treatment of acute asthma. Cochrane Database Syst Rev. 2017 Nov 28;11:CD003898.

17. Su Z, Li R, Gai Z. Intravenous and Nebulized Magnesium Sulfate for Treating Acute Asthma in Children: A Systematic Review and Meta-Analysis. Pediatr Emerg Care. 2018 Jun;34(6):390-395.

18. Modaresi MR, Faghihinia J, Kelishadi R, Reisi M, Mirlohi S, Pajhang F, Sadeghian M. Nebulized Magnesium Sulfate in Acute Bronchiolitis: A Randomized Controlled Trial. Indian J Pediatr. 2015 Sep;82(9):794-8.

19. Kose M, Ozturk MA, Poyrazoğlu H, Elmas T, Ekinci D, Tubas F, Kurt T, Goktas MA. The efficacy of nebulized salbutamol, magnesium sulfate, and salbutamol/magnesium sulfate combination in moderate bronchiolitis. Eur J Pediatr. 2014 Sep;173(9):1157-60.

20. Alansari K, Sayyed R, Davidson BL, Al Jawala S, Ghadier M. IV Magnesium Sulfate for Bronchiolitis: A Randomized Trial. Chest. 2017 Jul;152(1):113-119.

21. Malhi KASK, Zahoor F. Modified respiratory distress assessment instrument score, predictor of outcome of acute lower respiratory tract infections in children. Pak J Med Sci. 2013;7:529-532.

22. Guilbert TW, Morgan WJ, Zeiger RS, Bacharier LB, Boehmer SJ, Krawiec M, Larsen G, Lemanske RF, Liu A, Mauger DT, et al. Atopic characteristics of children with recurrent wheezing at high risk for the development of childhood asthma. J Allergy Clin Immunol 2004;114:1282-1287. 
23. Alansari K, Ahmed W, Davidson BL, Alamri M, Zakaria I, Alrifaai M. Nebulized magnesium for moderate and severe pediatric asthma: A randomized trial. Pediatr Pulmonol. 2015 Dec;50(12):11919.

\section{Hosted file}

Table 1.pdf available at https://authorea.com/users/357979/articles/510297-intravenousmagnesium-sulfate-for-acute-bronchiolitis-evaluation-of-the-effect-on-clinical-courseand-outcomes

\section{Hosted file}

Figure 1.pdf available at https://authorea.com/users/357979/articles/510297-intravenousmagnesium-sulfate-for-acute-bronchiolitis-evaluation-of-the-effect-on-clinical-courseand-outcomes

\section{Hosted file}

Figure 2.pdf available at https://authorea.com/users/357979/articles/510297-intravenousmagnesium-sulfate-for-acute-bronchiolitis-evaluation-of-the-effect-on-clinical-courseand-outcomes

\section{Hosted file}

Figure 3.pdf available at https://authorea.com/users/357979/articles/510297-intravenousmagnesium-sulfate-for-acute-bronchiolitis-evaluation-of-the-effect-on-clinical-courseand-outcomes 